\title{
INVESTIGACIÓN DEL COMPORTAMIENTO INFORMACIONAL A TRAVÉS DEL ANÁLISIS DE REDES SOCIALES
}

\author{
Aurora González-Teruel y Carolina Andreu-Ramos
}
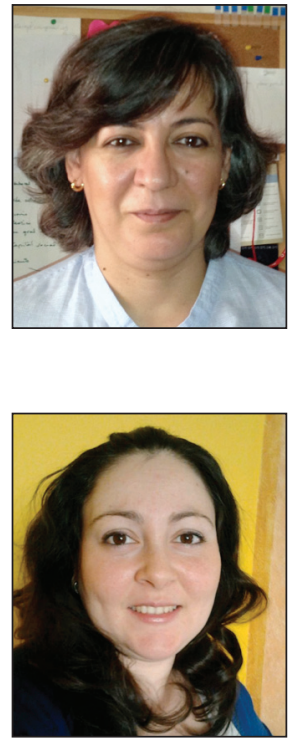

Aurora González-Teruel es profesora titular de biblioteconomía y documentación en el Departamento de Historia de la Ciencia y Documentación de la Universitat de València. Desarrolla la línea de investigación "Fundamentos teóricos y metodológicos del comportamiento informacional". Sobre este tema ha publicado trabajos en revistas nacionales e internacionales así como las monografías Los estudios de necesidades y usos de la información: fundamentos y perspectivas actuales (2005) y Métodos y técnicas para la investigación del comportamiento informacional: fundamentos y nuevos desarrollos (2012).

http://orcid.org/0000-0001-9304-2928

agonzal@uv.es

Carolina Andreu-Ramos es licenciada en periodismo y graduada en información y documentación, con experiencia como redactora de contenidos en diversos medios de comunicación y como gestora de información en general. Ha trabajado como consultora en estrategias de posicionamiento SEO en buscadores web y realizado distintas tareas como técnica documentalista en la migración de datos entre software de gestión bibliotecaria.

http://orcid.org/0000-0002-1411-6800

carolina.andreu.ramos@gmail.com

Universitat de València. Facultad de Medicina. Departamento de Historia de la Ciencia y Documentación Blasco Ibáñez, 15. 46010 Valencia, España

\section{Resumen}

El enfoque prevaleciente en la investigación del comportamiento informacional es el constructivista centrado en el individuo como generador de conocimiento. Recientemente han aumentado las críticas a esta postura reclamando un giro hacia lo social. Desde esta perspectiva se emplean métodos como el análisis del discurso que busca conocer, a través del lenguaje de los usuarios, cómo se genera y comparte el conocimiento en el contexto social. Otros métodos más objetivistas como el análisis de redes sociales podrían contribuir a un mayor conocimiento de este aspecto. Este trabajo describe cómo se ha aplicado a la investigación del comportamiento informacional, se presenta una aplicación práctica y se discuten las implicaciones profesionales y metodológicas.

\section{Palabras clave}

Comportamiento informacional, Análisis de redes sociales, Metodología de investigación, Diseño de la investigación, Práctica profesional.

\section{Title: Information behavior research through social network analysis}

\begin{abstract}
The field of information behaviour research is dominated by a constructivist vision focused on the individual as a generator of knowledge. Recently, a group of researchers have increased the criticism of this approach and advocate a social turn. From this point of view, methods such as discourse analysis are used to try to learn from the language of users how they generate and share knowledge in the social context. Other methods that could contribute to a better understanding of this aspect include social network analysis. The article describes how this method has been applied to the study of information behaviour, shows a practical application and discusses the professional and methodological implications.
\end{abstract}

\section{Keywords}

Information behaviour, Social network analysis, Research methodology, Research design, Professional practice.

González-Teruel, Aurora; Andreu-Ramos, Carolina. "Investigación del comportamiento informacional a través del análisis de redes sociales". El profesional de la información, noviembre-diciembre, v. 22, n. 6, pp. 522-528.

\section{http://dx.doi.org/10.3145/epi.2013.nov.04}




\section{Enfoque individual y social en la investigación del comportamiento informacional}

Se llama comportamiento informacional $(\mathrm{Cl})$ a cualquier experiencia humana relacionada con la necesidad, búsqueda, gestión, difusión y uso de la información en diferentes contextos (Fisher; Erdelez; McKechnie, 2005). Su observación persigue planificar sistemas de información adecuados para la satisfacción de los usuarios.

Teorías del $\mathrm{Cl}$ ampliamente seguidas y citadas, como el Sense-making (Dervin, 1983) o el Information-seeking process (Kuhlthau, 1993), se concibieron en su mayor parte bajo presupuestos constructivistas, un enfoque basado en que la realidad no es previa al conocimiento sino construida por el individuo a medida que es conocida por sus estructuras cognitivas (Santoianni; Striano, 2006). La consecuencia para la investigación del $\mathrm{Cl}$ ha sido que se ha enfatizado el papel del usuario como procesador de información, activo e imaginativo, puesto que éste no sólo recibe mensajes sino que crea o inventa activamente un sentido para ellos (Tuominen; Talja; Savolainen, 2002), es decir, él es el auténtico generador de conocimiento.

No obstante, más recientemente un grupo de investigadores, en el contexto del construccionismo social y/o la teoría crítica, han cuestionado esta perspectiva individualista proponiendo alternativas. Éstas hacen hincapié en la dimensión social, considerándola no como una variable más del $\mathrm{Cl}$ sino un componente esencial en el proceso de generación de conocimiento. Igualmente, han cuestionado la utilidad de las teorías constructivistas porque no son adecuadas para observar el intercambio de conocimiento en el contexto de las redes sociales (Olsson, 2012), no representan todas las experiencias posibles pues se centran únicamente en búsquedas intencionales o se basan en la observación de grupos privilegiados (investigadores o profesionales) y, consecuentemente, describen búsquedas sistemáticas muy diferentes a las de la vida cotidiana (Mckenzie, 2003).

Desde una perspectiva metodológica, consideran que los métodos empleados bajo el enfoque constructivista no posibilitan acceder al objeto central de su enfoque teórico, las estructuras cognitivas de los informantes, sino únicamente a la representación de la interacción social entre investigador e informante (Olsson, 2006). Así, se exploran métodos novedosos, como el análisis del discurso, orientados a identificar los medios por los cuales el conocimiento se genera y comparte dentro de un grupo social. Éste se basa en la idea de que el lenguaje revela las construcciones sociales del informante $y$, por lo tanto, para entender cómo genera e intercambia conocimiento se debe estudiar su discurso, en lugar de limitarse a observar su comportamiento (Courtright, 2008).

Junto con los métodos de corte interpretativo, el análisis de redes sociales (ARS) puede enriquecer el proceso de investigación pues lo orienta a la observación de la generación e intercambio de conocimiento en contextos sociales. Como afirma Rodríguez (2005), el ARS va más allá de una investigación individualista que ignora a otros actores pues explica el comportamiento de los individuos como resultado de su participación en relaciones sociales estructuradas.

\section{El comportamiento informacional desde el punto de vista de la estructura social}

Una red social es una estructura compuesta por nodos o actores, algunos de los cuales están conectados por relaciones o vínculos (Knoke; Yang, 2008). La mayor diferencia entre los datos convencionales y los reticulares es que los primeros se centran en actores y atributos mientras los segundos en actores y relaciones. Por ello orienta la observación del comportamiento informacional ( $\mathrm{Cl}$ ) hacia el usuario (el nodo de una red) en función de un grupo social (el conjunto de relaciones) más que hacia el individuo influido, entre otros, por factores sociales.

El uso del análisis de redes sociales (ARS) en Documentación ha aumentado en los últimos años (Schultz-Jones, 2009) aunque centrado en las redes de publicación, citación o colaboración científica, así como en redes sociales online como Facebook. En el contexto del $\mathrm{Cl}$, Haythornthwaite (1996) describió su potencial para la observación del intercambio de información; sin embargo, actualmente no es una de las metodologías más utilizadas (González-Teruel; Barrios-Cerrejón, 2012). Pese a las pocas investigaciones que lo utilizan, su análisis proporciona una visión amplia de sus posibilidades (tabla 1 ).

\section{El ARS posibilita la observación del usua- rio (nodo) en función de un grupo social (vínculos) más que hacia el individuo in- fluido por factores sociales}

El ARS se ha empleado para la observación del Cl tanto en el contexto organizacional como en el de la vida cotidiana. Así, para el primer caso es posible encontrar, por ejemplo, investigaciones que evidencian los flujos formales e informales de información, su frecuencia y canal por el que se producen (Haythornthwaite; Wellman, 1998) o los criterios para elegir a una persona como fuente de información (Mackenzie, 2005). En el contexto de la vida cotidiana, el ARS ha permitido abordar propósitos que la investigación tradicional sobre usuarios nunca había considerado. Así Hersberger (2003), por ejemplo, estudia el intercambio de información en redes de poblaciones con riesgo de exclusión como los sin-techo (homeless) o Veinot (2010) en redes de personas con peligro de estigmatización como enfermos de VIH/SIDA.

Además, la obtención de la red se aborda desde dos puntos de vista:

- Primero a partir de las personas del entorno de un individuo con las que dice intercambiar información. Se trata de la red personal o egocéntrica que es la que rodea a un nodo, conocido como ego. Es el caso del análisis de Laplante (2011) en el que se evidencian los canales interpersonales por los que los adolescentes descubren nueva música.

- En segundo lugar, la red completa en la que, a partir de las personas de un entorno, se pide al informante que elija aquellas con las que intercambia información. Se representa una estructura social delimitada en vez de sólo los 
Tabla 1

\begin{tabular}{|c|c|c|c|c|c|c|}
\hline & Contexto & Usuario & Aspecto $\mathrm{Cl}$ & Tipo de red & Técnica & ARS \\
\hline Courtright (2005) & $\begin{array}{l}\text { Vida cotidiana- } \\
\text { salud }\end{array}$ & Emigrantes & $\begin{array}{l}\text { Búsqueda de infor- } \\
\text { mación en salud }\end{array}$ & Egocéntrica & Entrevistas & $\begin{array}{l}\text { Composición de la red, } \\
\text { fuerza de los vínculos, } \\
\text { papel de las instituciones }\end{array}$ \\
\hline $\begin{array}{l}\text { Haythornthwaite } \\
\text { y Wellman (1998) }\end{array}$ & $\begin{array}{l}\text { Organizacional- } \\
\text { académico-inves- } \\
\text { tigación }\end{array}$ & Investigadores & $\begin{array}{l}\text { Relaciones y medio } \\
\text { para el intercambio } \\
\text { de información }\end{array}$ & Completa & Cuestionario & $\begin{array}{l}\text { Frecuencia, medio y } \\
\text { contenido }\end{array}$ \\
\hline Hersberger (2003) & Vida cotidiana & Homeless & Red apoyo social & Egocéntrica & Entrevista & $\begin{array}{l}\text { Composición de la red, } \\
\text { tamaño, capital social }\end{array}$ \\
\hline Johnson (2007) & Vida cotidiana & Ciudadanos & $\begin{array}{l}\text { Modo de obtener } \\
\text { información/capital } \\
\text { social }\end{array}$ & Egocéntrica & Cuestionario & $\begin{array}{l}\text { Tamaño de la red, } \\
\text { densidad, intensidad, } \\
\text { diversidad y alcance } \\
\text { (capital social) }\end{array}$ \\
\hline Laplante (2011) & Vida cotidiana & Adolescentes & $\begin{array}{l}\text { Intercambio de } \\
\text { información sobre } \\
\text { música }\end{array}$ & Egocéntrica & $\begin{array}{l}\text { Entrevista/cues- } \\
\text { tionario }\end{array}$ & $\begin{array}{l}\text { Propiedades de los } \\
\text { actores, naturaleza de la } \\
\text { relación, cercanía, dura- } \\
\text { ción y frecuencia }\end{array}$ \\
\hline Mackenzie (2005) & $\begin{array}{l}\text { Organizacional- } \\
\text { empresa }\end{array}$ & Line-managers & $\begin{array}{l}\text { Criterios para elegir } \\
\text { una persona como } \\
\text { fuente de informa- } \\
\text { ción }\end{array}$ & Completa & Entrevista & $\begin{array}{l}\text { Dirección y frecuencia de } \\
\text { los vínculos, razones para } \\
\text { la selección }\end{array}$ \\
\hline Marouf (2007) & $\begin{array}{l}\text { Organizacional- } \\
\text { empresa }\end{array}$ & $\begin{array}{l}\text { Trabajadores em- } \\
\text { presa financiera }\end{array}$ & $\begin{array}{l}\text { Relación entre } \\
\text { la fuerza de los } \\
\text { vínculos y compartir } \\
\text { conocimiento }\end{array}$ & Completa & Cuestionario & $\begin{array}{l}\text { Frecuencia de interaccio- } \\
\text { nes, cercanía y densidad }\end{array}$ \\
\hline Morey (2006) & Vida cotidiana & Afroamericanos & $\begin{array}{l}\text { Acceso a informa- } \\
\text { ción en salud }\end{array}$ & Egocéntrica & Cuestionario & $\begin{array}{l}\text { Componentes de la red, } \\
\text { propiedades y fuerza de } \\
\text { los vínculos }\end{array}$ \\
\hline Pettigrew (1999) & $\begin{array}{l}\text { Vida cotidiana- } \\
\text { salud }\end{array}$ & Ancianos & $\begin{array}{l}\text { Flujos de informa- } \\
\text { ción entre ancianos } \\
\text { y personal de } \\
\text { enfermería }\end{array}$ & Egocéntrica & $\begin{array}{l}\text { Observación/ } \\
\text { entrevistas }\end{array}$ & Fuerza de los vínculos \\
\hline Veinot (2010) & $\begin{array}{l}\text { Vida cotidiana- } \\
\text { salud }\end{array}$ & $\begin{array}{l}\text { Enfermo de VIH/ } \\
\text { SIDA y su red }\end{array}$ & $\begin{array}{l}\text { Intercambio de in- } \\
\text { formación y ayuda }\end{array}$ & Egocéntrica & Entrevista & $\begin{array}{l}\text { Composición, tamaño y } \\
\text { capital social }\end{array}$ \\
\hline $\begin{array}{l}\text { Yousefi-Nooraie } \\
\text { et al. (2012) }\end{array}$ & $\begin{array}{l}\text { Organizacional- } \\
\text { sanitario }\end{array}$ & $\begin{array}{l}\text { Personal de } \\
\text { departamento de } \\
\text { salud pública }\end{array}$ & $\begin{array}{l}\text { Búsqueda de } \\
\text { información para las } \\
\text { decisiones basadas } \\
\text { en la práctica }\end{array}$ & Completa & Cuestionario & $\begin{array}{l}\text { Densidad, reciprocidad, } \\
\text { centralidad y brokerage }\end{array}$ \\
\hline
\end{tabular}

nodos que rodean el ego y es el caso de cualquiera de investigaciones del ámbito organizacional de la tabla 1.

\section{En el contexto de la vida cotidiana, el ARS ha permitido abordar propósitos que la investigación tradicional sobre usuarios nunca había considerado}

Para Haythornthwaite (1996) la red egocéntrica posibilita modelar el proceso de búsqueda para guiar a usuarios con necesidades de información similares, emprender cambios en un sistema de información para ajustarlo a la conducta del usuario o promocionar servicios que no estén siendo utilizados. Mientras que la red completa ayuda a identificar grupos de personas con tareas similares y consecuentemente necesidades de información similar, detectar individuos centrales en la provisión de información así como la posición de la unidad de información y detectar áreas mal provistas en las que la información se está necesitando.
Para ambos tipos de enfoques, Knoke y Yang (2008) describen procedimientos para identificar las personas de una red, normalmente con cuestionarios y entrevistas. Por ejemplo, Marouf (2007) presenta una lista completa de los actores que pueden formar parte de la red del informante, todas las personas de una organización, y éste señala aquellos con los que comparte información. Mientras que en el trabajo de Yousefi-Nooraie et al., (2012) el informante nombra cinco personas con las que realiza este intercambio. Además, en el contexto de la teoría del capital social, Johnson (2007) utiliza el generador de posición mediante el cual el informante señala a personas conocidas de una estructura social determinada. Las relaciones sociales entre los actores de la red y las posiciones a las que puedan acceder, determinarán su capital social.

Junto con los anteriores procedimientos, otros utilizan enfoques cualitativos menos estructurados. Así, Courtright (2005) elabora la red social mediante el análisis de contenido de las respuestas a entrevistas. Mientras que Pettigrew (1999) y Hersberger (2003) la obtienen presentando una serie de círculos concéntricos divididos en cuatro cuadran- 
tes que son amigos, familiares, vecinos y otros. En dichos círculos señalan las personas de la red mediante puntos, más o menos cercanos al centro (ego), y después dibujan los vínculos mediante líneas.

Por último, respecto a las medidas aplicadas al análisis de las redes, éstas son diversas y pueden centrarse en vínculos, propiedades estructurales 0 posiciones en la red. Con relación a los vínculos, es interesante la aplicación de la teoría de la fuerza

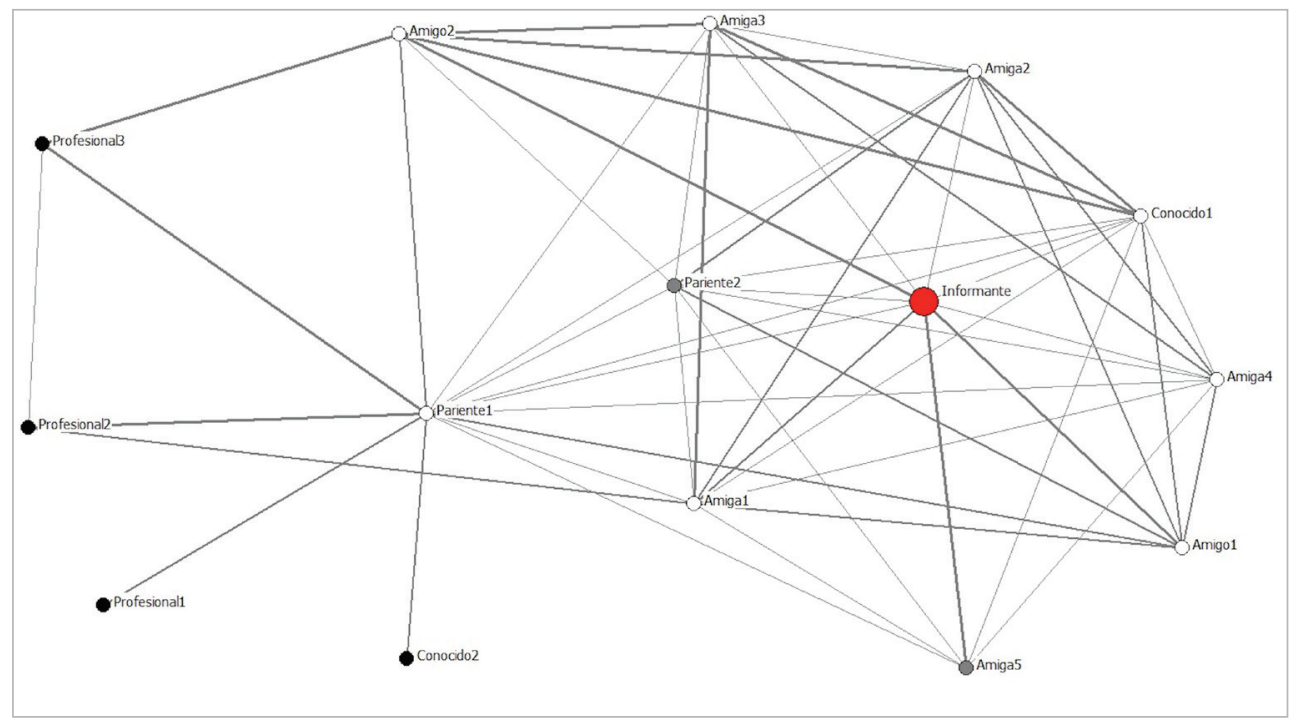

Figura 1. Red social de la informante 6 de los vínculos débiles de Granovetter (1973) según la cual la red social de un individuo está formada por vínculos fuertes y débiles. No obstante, sólo estos últimos servirán como enlaces a otra red social y permitirán acceder a información que no circula a través de los vínculos fuertes de la propia red. Para el análisis del $\mathrm{Cl}$, por ejemplo, Morey (2006) destaca la relevancia de los profesionales sanitarios (vínculos débiles) como proveedores de información en salud a los afroamericanos, o Pettigrew (1999) para el caso de los enfermeros en la red de los ancianos a los que asisten.

Respecto a las propiedades estructurales, las medidas más básicas son tamaño y densidad. Así el tamaño es el número de actores que forman parte de la red considerándose que, cuanto más grande, a más fuentes de información se tiene acceso y más variadas son. En el trabajo de Johnson (2007), por ejemplo, el tamaño medio de la red personal de los ciudadanos mongoles era de 14 personas. Aunque para valorar esta cifra es necesario compararla con investigaciones realizadas en contextos similares. En cuanto a la densidad, es la cantidad de vínculos existentes frente a los posibles. La información fluirá más rápidamente por redes altamente interconectadas, no obstante en una red con alta densidad y pocos vínculos a redes externas la información fluirá recurrentemente.

Por último, en cuanto al análisis de las posiciones dentro de una red, es interesante destacar el brokerage o el papel de conexión entre grupos de un actor, estudiado por YousefiNooraie et al., (2012) o la diversidad y el alcance investigado por Johnson (2007) como medidas del capital social de sus informantes.

\section{Comportamiento informacional de mujeres jubiladas viudas}

Para probar la utilidad del ARS, se diseñó una investigación exploratoria del $\mathrm{Cl}$ de las mujeres jubiladas viudas pues se consideró un sector con riesgo de exclusión informacional dada su dificultad para acceder a fuentes de información formales. Su realizó un muestreo teórico (Glaser; Strauss, 1967) y, conforme a ello, la incorporación de nuevas informantes finalizó cuando más datos resultaban redundantes.

La muestra final estuvo compuesta por ocho mujeres de entre 75 y 85 años, la mayor parte vivía sola, sin estudios o estudios primarios $y$, en todos los casos excepto en uno, habían tenido un oficio o varios durante su vida.

\section{Desempeñar una profesión basada en la provisión de servicios de información sin un conocimiento sistemático de los usuarios aleja del papel central que se pretende ejercer en ese medio}

Para la recogida de datos se empleó una entrevista semiestructurada y un cuestionario. Previamente firmaron un consentimiento informado. Con la entrevista se buscaba obtener datos generales sobre su $\mathrm{Cl}$. Mediante el cuestionario se recabó información sobre la red social de cada informante. Los datos obtenidos a través de las entrevistas se analizaron por medio de un análisis de contenido cualitativo generando categorías descriptivas del $\mathrm{Cl}$ de esta población y permitiendo la contextualización de las redes sociales de las mujeres entrevistadas. Los obtenidos a través del cuestionario fueron la representación gráfica de la red así como su tamaño. Finalmente, para asegurar la calidad del proceso, se siguieron dos de los criterios de Lincoln y Guba (1985), la credibilidad mediante la revisión de los resultados por un investigador externo y la confirmabilidad por medio del registro de la documentación producida en una auditoría de toda la secuencia del proceso (audit-trail).

Del análisis se obtuvieron dos tipos de resultados. Primero las categorías que describían el proceso de búsqueda de información y, en concreto, problemas informativos con los que se enfrentaban, estrategias para su resolución, modo de acceder a la información y modo de resolver situaciones que motivaban ese proceso de búsqueda. Además, algunos de los resultados de las entrevistas permitieron contextualizar las redes sociales. Por ejemplo, a pesar de que en principio las mujeres nombraban los medios de comunicación 


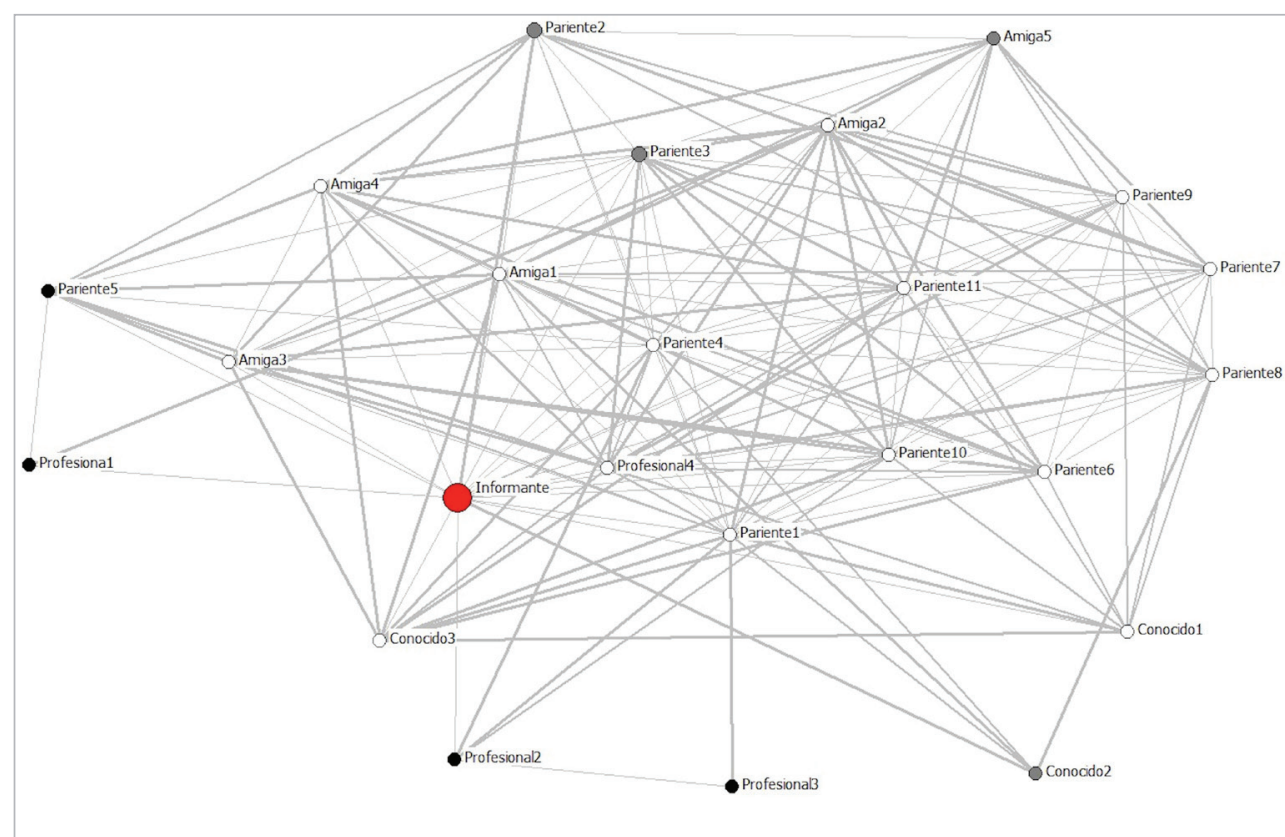

Figura 2. Red social de la informante 1

como medio preferente para acceder a la información, de análisis detallado se vio la importancia atribuida a sus redes personales para este propósito, una diferencia basada en la confianza y autoridad. Por otra parte, preguntadas sobre el uso de la biblioteca pública, en todos los casos excepto en uno no la habían utilizado por su limitado nivel de alfabetización y considerarla únicamente como prestadora de libros.

\section{Es necesario estudiar al usuario pero también lo es el que su investigación se oriente a la acción}

El segundo tipo de resultados fue el del cuestionario referente a la red de cada informante. Así, se obtuvo que el tamaño medio era de 19 nodos, la red con menos actores fue de 13 nodos y la que más de 27 . En cuanto a su composición, en primer lugar, estaban los familiares más directos seguidos por amigas a quienes normalmente les unía un vínculo de más de diez años. Por detrás de éstas, se encontraban otras personas a quienes habían conocido por motivos profesionales o porque eran trabajadores de empresas de cuyos servicios hicieron uso durante muchos años (ej., trabajadores del banco), e incluso porque habían contactado con ellos mediante familiares y amigos sin ser una relación continuada.

La figura 1 representa una de las redes más pequeñas con 15 nodos. Pertenece a una persona de 77 años, sin estudios, con capacidad de lectura y escritura limitada que durante su vida trabajó en la agricultura y como empleada del hogar. Los vínculos directos más estrechos son con amigos-as a los que conoce hace más de diez años, casi todos sin estudios o con estudios primarios. Los vínculos que tiene con personas con estudios universitarios (profesionales y/o conocidos) no son directos y los tiene a través de los primeros.
La figura 2 presenta una de las redes con más nodos, 24. Es de una persona con circunstancias similares a la anterior con la única diferencia que durante su vida tuvo más trabajos en diferentes entornos. En este caso se trata de una red con mayor capital social, más y más variados vínculos. Los directos no sólo se establecen con familiares y/o amigos sino también con profesionales y conocidos con los que tuvo un contacto puntual. Por otra parte, el número de actores con estudios universitarios es mayor. Algunas situaciones narradas durante las entrevistas por las informantes con redes mayores, muestran que todos estos vínculos les han servido para acceder a información de vital importancia, como por ejemplo la de un mejor empleo. No obstante, también describen como, tras quedar viudas, los familiares directos y particularmente los hijos, se convierten en el centro de sus redes sociales, siendo éstos los nodos que generalmente les abren las puertas a otros vínculos.

\section{Implicaciones para la investigación del comportamiento informacional}

A pesar de lo limitado de los resultados aquí presentados, se pueden extraer implicaciones profesionales y metodológicas.

La principal crítica a la investigación sobre comportamiento informacional $(\mathrm{Cl})$ es que los resultados que genera son esperables o no aplicables a la mejora de los sistemas de información. Sin embargo, la planificación y evaluación de esos sistemas y, en general, desempeñar una profesión basada en la provisión de servicios de información sin un conocimiento sistemático del medio humano con el que se interactúa, aleja del papel central que se pretende ejercer en ese medio. La manida frase "satisfacción del usuario" se vacía de contenido y únicamente deja entrever la autocomplacencia que, con la seguridad que dan unos conocimientos y capacidades técnicas, lleva a asumir una posición de poder desigual hacia un usuario necesitado de ayuda o asesoramiento. Sin embargo, aunque las mujeres jubiladas viudas no utilicen fuentes de información formales, incluida la biblioteca pública, no por ello dejan de resolver sus problemas cotidianos. Tienen sus propios medios, su red social, que aunque pequeña o limitada en cuanto a capital social, les ha demostrado ser útil, eficiente y confían en ella. Quizá sea el momento en el que el profesional de la información se integre en esa red contribuyendo a aumentar su capital social.

Así, el ARS sitúa el diseño de la investigación en un terreno al que tradicionalmente no había llegado, por carencias con- 
ceptuales o metodológicas o por desinterés por poblaciones distintas a científicos o profesionales. En la vida cotidiana, tal y como evidencia en el caso aquí presentado, los problemas de información no dejan de resolverse. Y es precisamente en este contexto donde quizá la investigación del $\mathrm{Cl}$ sea más necesaria para favorecer un acceso equitativo a la información. Pero una investigación que, más allá de ejercicios académicos, oriente el diseño hacia la obtención de resultados aplicables a la mejora de los sistemas de información. Y para ello es necesario una conceptualización clara y real de las cuestiones a resolver y una metodología que, como la que aquí se presenta, permita la descripción del medio social a través del cual se genera y comparte conocimiento pero también la comprensión de los mecanismos que llevan a ello.

\section{Conclusión}

El enfoque constructivista de la investigación del comportamiento informacional ( $\mathrm{Cl}$ ), y con ello la concepción de diversas teorías, aportó más rigor y una profunda reflexión sobre el objeto de esta investigación. Más recientemente surgieron nuevas estrategias para abordar dicha investigación haciendo hincapié en la observación del usuario en función de su mundo social. A pesar de que en ocasiones se presentan como posturas contrapuestas, incluso identificándose con denominaciones distintas (comportamiento informacional vs prácticas de información), ambas abren interesantes posibilidades para el conocimiento del usuario. No obstante, más allá de sus presupuestos ontológicos o epistemológicos, es necesario un mayor esfuerzo en el diseño de investigaciones que aborden cuestiones reales, empleen metodologías adecuadas y expongan claramente las implicaciones prácticas de sus resultados.

\section{Bibliografía}

Courtright, Christina (2005). "Health information-seeking among latino newcomers: an exploratory study". Information research, v. 10, n. 2.

http://informationr.net/ir/10-2/paper224.html

Courtright, Christina (2008). "Context in information behavior research". Annual review of information science and technology, v. 41, pp. 273-306.

http://dx.doi.org/10.1002/aris.2007.1440410113

Dervin, Brenda (1983). "An overview of sense-making research: concepts, methods, and results to date". En: Annual meeting of the International Communication Association.

Fisher, Karen; Erdelez, Sanda; McKechnie, Lynne (2005). Theories of information behavior. Medford: Information Today. ISBN: 9781573872300

Glaser, Barney; Strauss, Anselm (1967). The discovery of grounded theory. Strategies for qualitative research. Chicago: Aldine De Gruyter. ISBN: 9780202300283

González-Teruel, Aurora; Barrios-Cerrejón, Maite (2012). Métodos y técnicas para la investigación del comportamiento informacional: fundamentos y nuevos desarrollos. Gijón: Trea. ISBN: 9788497046718

Granovetter, Mark (1973). "The strength of weak ties".
American journal of sociology, v. 78, n. 6, pp. 1360-1380. http://dx.doi.org/10.1086/225469

Haythornthwaite, Caroline (1996). "Social network analysis: an approach and technique for the study of information exchange". Library \& information science research, v. 18, n. 4, pp. 323-342.

http://dx.doi.org/10.1016/S0740-8188(96)90003-1

Haythornthwaite, Caroline; Wellman, Barry (1998). "Work, friendship, and media use for information exchange in a networked organization". Journal of the American Society for Information Science, v. 49, n. 12, pp. 1101-1114.

http://dx.doi.org/10.1002/(SICI)1097-4571(1998)49:12<1101::AIDASI6>3.3.CO;2-S

Hersberger, Julie (2003). "A qualitative approach to examining information transfer via social networks among homeless populations". The new review of information behaviour research, v. 4, n. 1, pp. 95-108.

http://dx.doi.org/10.1080/14716310310001631462

Johnson, Catherine (2007). "Social capital and the search for information: examining the role of social capital in information-seeking behavior in Mongolia". Journal of the American Society for Information Science and Technology, v. 58, n. 6, pp. 883-894.

http://dx.doi.org/10.1002/asi.20561

Knoke, David; Yang, Song (2008). Social network analysis. Thousands Oaks: Sage. ISBN: 9781412927499

Kuhlthau, Carol (1993). Seeking meaning: a process approach to library and information services. Norwood: Ablex. ISBN: 9780893919689

Laplante, Audrey (2011). "Social capital and music discovery: an examination of the ties through wich late adolescents discover new music". En: $12^{\text {th }}$ International Society for Music Information Retrieval Conf., pp. 341-346.

Lincoln, Yvonna; Guba, Egon (1985). Naturalistic inquiry. Beverly Hills: Sage. ISBN: 9780803924314

Marouf, Laila (2007). "Social networks and knowledge sharing in organizations: a case study". Journal of knowledge management, v. 11, n. 6, pp. 110-125.

http://dx.doi.org/10.1108/13673270710832208

Mckenzie, Maureen (2005). "Managers look to the social network to seek information". Information research, v. 10, n. 2. http://informationr.net/ir/10-2/paper216.htm/

McKenzie, Pamela (2003). "A model of information practices in accounts of everyday-life information-seeking". Journal of documentation, v. 59, n. 1, pp. 19-40. http://dx.doi.org/10.1108/00220410310457993

Morey, Ophelia (2006). "Health information ties: preliminary findings on the health information-seeking behaviour of an African-American community". Information research, v. 12, n. 2. http://informationr.net/ir/12-2/paper297.html

Olsson, Michael (2006). “Beyond 'needy' individuals: conceptualizing information behavior". Procs of the American Society for Information Science and Technology, v. 42, n. 1, pp. 43-55. http://dx.doi.org/10.1002/meet.1450420161 
Olsson, Michael (2012). "Ciphers to this great accompt. The Shakespearian social sense-making of theatre professionals". En: Widén-Wulff, Gunilla; Holmberg, Kim. Social information research. Bingley: Emerald Group, pp. 17-42. ISBN: 9781780528328

http://dx.doi.org/10.1108/S1876-0562(2012)0000005004

Pettigrew, Karen (1999). "Waiting for chiropody: contextual results from an ethnographic study of the information behaviour among attendees at community clinics". Information processing \& management, v. 35, n. 6, pp. 801-817. http://dx.doi.org/10.1016/S0306-4573(99)00027-8

Rodríguez, Josep (2006). Análisis estructural y de redes. Madrid: CIS. ISBN: 9788474762242

Santoianni, Flavia; Striano, Maura (2006). Modelos teóricos y metodológicos de la enseñanza. México: SigloXXI. ISBN: 9789682326202

Schultz-Jones, Barbara (2009). "Examining information be- havior through social networks: an interdisciplinary review". Journal of documentation, v. 65, n. 4, pp. 592-631.

http://dx.doi.org/10.1108/00220410910970276

Tuominen, Kimmo; Talja, Sanna; Savolainen, Reijo (2002). "Discourse, cognition, and reality: toward a social constructionist metatheory for LIS". En: CoLIS4. Procs of the Fourth int conf on conceptions of LIS, pp. 271-283.

Veinot, Tiffany (2010). "A multilevel model of HIV/AIDS information/help network development". Journal of documentation, v. 66, n. 6, pp. 875-905.

http://dx.doi.org/10.1108/00220411011087850

Yousefi-Nooraie, Reza; Dobbins, Maureen; Brouwers, Melissa; Wakefield, Patricia (2012). “Information-seeking for making evidence-informed decisions: a social network analysis on the staff of a public health department in Canada". BMC Health services research, v. 12:118. http://dx.doi.org/10.1186/1472-6963-12-118

\section{EmeraldeJournals}

\section{Más de 180.000 artículos, ofreciendo una cobertura completa de las disciplinas de gestión y temas complementarios especializados, incluso...}

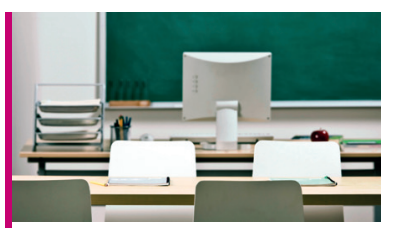
es un gran aliado de la comunidad de docentes como editorial de la publicación oficial del University Vocational Awards Council, Higher Education, Skills and Work-based Learning, y patrocina los premios UCEA Roald F. Campbell Lifetime Achievement Award (premio a los logros conseguidos a lo largo de toda una trayectoria profesional) y ASHE Best Dissertation Award (premio a la mejor tesis doctoral).

Los títulos más destacados son Education + Training, que lleva 55 años publicándose, y el Journal of Educational Administration, que ha publicado artículos de prominentes académicos de prestigiosas instituciones, como las Universidades de Stanford, Vanderbilt y la Universidad Estatal de Pensilvania.

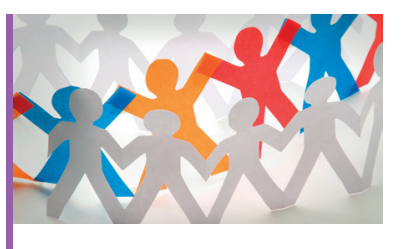

Recursos Humanos, Aprendizaje \& Estudios Organizacionales

Abarca las disciplinas más relevantes, incluidas el aprendizaje y el desarrollo, la gestión y medición del rendimiento, los estudios organizacionales y la gestión de los recursos humanos. Los autores son académicos de prestigiosas instituciones del mundo entero, como la Universidad de Harvard (EE. UU.), Cass Business School (Reino Unido), la Universidad de Stanford (EE. UU.) y la Universidad de Cambridge (Reino Unido).

La colección comprende títulos que figuran en el índice de Thomson Reuters (ISI), incluyendo el Journal of Organizational Change Management y el Journal of Managerial Psychology, con un factor de impacto de 1.179.

\section{Póngase en contacto con nosotros}

Si necesita que respondan sus preguntas con rapidez o desea hablar sobre las opciones de compra que mejor se adaptarían a sus necesidades, póngase en contacto con nosotros en nuestra oficina principal en el Reino Unido: Emerald Group Publishing Limited, Howard House, Wagon Lane, Bingley, BD16 1WA 